\title{
New results on the equivalence between zero-one programming and continuous concave programming
}

\author{
Francesco Rinaldi
}

Received: 5 February 2009 / Accepted: 16 February 2009 / Published online: 5 March 2009

(C) Springer-Verlag 2009

\begin{abstract}
In this work, we study continuous reformulations of zero-one concave programming problems. We introduce new concave penalty functions and we prove, using general equivalence results here derived, that the obtained continuous problems are equivalent to the original combinatorial problem.
\end{abstract}

Keywords Zero-one programming · Concave functions · Continuous programming · Exact penalty functions

\section{Introduction}

In this article, we consider the zero-one programming problem:

$$
\begin{aligned}
& \min f(x) \\
& x \in P \\
& x \in\{0,1\}^{n}
\end{aligned}
$$

where:

(1) $f: R^{n} \rightarrow R$ is a concave function;

(2) $P \subset R^{n}$ is a polyhedral set.

We are interested in studying equivalent continuous reformulations of problem (1), where the zero-one constraints on the variables are relaxed, and the objective function

F. Rinaldi $(\bigotimes)$

Istituto di Analisi dei Sistemi ed Informatica,

Consiglio Nazionale delle Ricerche,

Viale Manzoni 30, 00185 Rome, Italy

e-mail: francesco.rinaldi@iasi.cnr.it 
contains terms penalizing the violation of such constraints. A well-known continuous formulation equivalent to (1) was introduced in [4] for a more general class of problems, and takes the form

$$
\begin{aligned}
& \min f(x)+\frac{1}{\beta} \sum_{i=1}^{n} x_{i}\left(1-x_{i}\right) \\
& x \in P \\
& 0 \leq x \leq e
\end{aligned}
$$

where $e \in R^{n}$ is the vector of ones, and $\beta>0$ is the penalty parameter. Other important contributions about the transformation of discrete optimization problems into equivalent continuous problems can be found in [3,5-7,10-12].

Note that the employment of reformulations of a given (possibly mixed) integer problem in a continuous space can be a practicable technique for finding good solutions even when dealing with large-scale problems (see e.g., $[1,2,8,9,13])$.

The aim of this work is to introduce new continuous formulations equivalent to (1). These formulations take the common form

$$
\begin{aligned}
& \min f(x)+g(x, u) \\
& x \in P \\
& 0 \leq x \leq e
\end{aligned}
$$

where $g(\cdot, u): R^{n} \rightarrow R$ is a concave function depending on a vector of parameters $u \in U \subseteq R^{m}$. We remark that a wider availability of equivalent formulations is important since it can facilitate, in connection with the adoption of suitable global optimization methods, the search of optimal solutions for different classes of boolean problems. However, this work has theoretical aims, so that algorithmic and computational issues are not investigated, but they can be the object of a future work on specific combinatorial problems.

The article is organized as follows. We first derive general results on the equivalence between an unspecified optimization problem and a parameterized family of problems. Then, with reference to the specific problem (1), we introduce four new penalty functions. Thanks to the equivalence results, we easily prove that the problems of the form (3) related to the proposed penalty functions are equivalent to the original zero-one programming problem.

\section{Results on the equivalence between problems}

In this section we state general results on the equivalence between problems. Consider the problem:

$$
\min _{x \in X \cap T} h(x)
$$


where $T \subset R^{n}, X \subset R^{n}$ and $h: R^{n} \rightarrow R$ is bounded on T. Assume that problem (4) admits solutions. Let $g(\cdot, u): R^{n} \rightarrow R$ be a function depending on a vector of parameters $u \in U \subseteq R^{m}$.

For any $u \in U$, consider the following problem

$$
\min _{x \in W \cap T} h(x)+g(x, u)
$$

where $W$ is a set such that $X \subseteq W \subset R^{n}$. For instance, considering (1) as problem (4), and (2) as problem (5), we have:

$-T=P$

$-\quad X=\{0,1\}^{n}$

- $W=\left\{x \in R^{n}: 0 \leq x_{i} \leq 1, i=1, \ldots, n\right\}$;

- $u=1 / \beta, g(x, u)=\frac{1}{\beta} \sum_{i=1}^{n} x_{i}\left(1-x_{i}\right)$.

Before we define and prove general equivalence results, we state the following assumptions.

Assumption 1 There exists a finite set $S^{\star}$ having the property that, for any $u \in U, a$ point $x(u) \in S^{\star}$ exists such that

$$
x(u) \in \min _{x \in W \cap T} h(x)+g(x, u) .
$$

Assumption 2 There exists an infinite sequence $\left\{u^{k}\right\} \subset U$ such that

(i) $\forall x, y \in X$

$$
g\left(x, u^{k}\right)=g\left(y, u^{k}\right)
$$

(ii) $\forall \tilde{x} \notin X, \forall \bar{x} \in X$

$$
\lim _{k \rightarrow \infty}\left[g\left(\tilde{x}, u^{k}\right)-g\left(\bar{x}, u^{k}\right)\right]=+\infty
$$

General conditions sufficient to ensure the equivalence between (4) and (5) are given in the following theorem.

Theorem 1 Suppose that Assumption 1 holds, and let $\left\{u^{k}\right\} \subset U$ be an infinite sequence satisfying Assumption 2. Then, for $k$ sufficiently large problem (5) with $u=u^{k}$ and problem (4) have the same minimum points.

Proof The proof is divided in two parts.

(Part I). Let $x^{\star}$ be an optimal solution of problem (4). By contradiction, assume there exists a subsequence $\left\{u^{k}\right\}_{K}$ such that, for all $k \in K$, denoting by $x^{k} \in S^{\star}$ a solution of (5), we have

$$
h\left(x^{k}\right)+g\left(x^{k}, u^{k}\right)<h\left(x^{\star}\right)+g\left(x^{\star}, u^{k}\right) .
$$


Since $x^{k} \in S^{\star}$ and $S^{\star}$ is finite, then we can extract a further subsequence such that $x^{k}=\tilde{x}$ for all $k \in K$, and hence, from (7), it is possible to write

$$
h(\tilde{x})+g\left(\tilde{x}, u^{k}\right)<h\left(x^{\star}\right)+g\left(x^{\star}, u^{k}\right) .
$$

We now consider two different cases

(1) $\tilde{x} \in X:$ By (i) of Assumption 2 and inequality (8), we obtain

$$
h(\tilde{x})<h\left(x^{\star}\right),
$$

but this contradicts the fact that $x^{\star}$ is optimum for problem (4).

(2) $\tilde{x} \notin X$ : By using inequality (8) and boundedness of $f$, we have

$$
g\left(\tilde{x}, u^{k}\right)-g\left(x^{\star}, u^{k}\right)<h\left(x^{\star}\right)-h(\tilde{x}),
$$

but this contradicts ( $i i)$ of Assumption 2 .

(Part II). Let $\left\{x^{k}\right\}$ be the sequence of optimal solutions of problem (5) related to the sequence $\left\{u^{k}\right\}$, with $x^{k} \in S^{\star}$ for all $k$. By contradiction, assume there exists a solution $\bar{x}$ of problem (4) such that

$$
h(\bar{x})<h\left(x^{k}\right) .
$$

By optimality of $x^{k}$, we have

$$
h\left(x^{k}\right)+g\left(x^{k}, u^{k}\right) \leq h(\bar{x})+g\left(\bar{x}, u^{k}\right) .
$$

Since $x^{k} \in S^{\star}$ and $S^{\star}$ is finite, then we can extract a further subsequence such that $x^{k}=\tilde{x}$ for all $k \in K$. Once again, we consider two different cases:

(1) $\tilde{x} \in X$ : By ( $i$ ) of Assumption 2 and inequality (11), we obtain

$$
h(\tilde{x}) \leq h(\bar{x})
$$

but this contradicts (10).

(2) $\tilde{x} \notin X$ : By using inequality (11) and boundedness of $h$, we have

$$
g\left(\tilde{x}, u^{k}\right)-g\left(\bar{x}, u^{k}\right) \leq h(\bar{x})-h(\tilde{x}),
$$

but this contradicts (ii) of Assumption 2.

Using Theorem 1 we derive equivalence results for problems having a concave penalty formulation. First we recall a well-known result (see Corollary 32.3.4 in [14]) concerning the minimization of a concave function over a polyhedral set:

Proposition 1 Let $\xi$ be a concave function, and let $C$ be a nonempty polyhedral convex set contained in dom $\xi$. Suppose that $C$ contains no lines, and that $\xi$ is bounded below on $C$. Then the infimum of $\xi$ relative to $C$ is attained at one of the (finitely many) vertices of $C$. 
Proposition 2 Suppose that the feasible set of problem (5) is a polyhedral set, and, for any $u \in U$, the objective function is concave and bounded below on the feasible set. Let $\left\{u^{k}\right\} \subset U$ be an infinite sequence such that Assumption 2 is satisfied. Then, for $k$ sufficiently large problem (5) with $u=u^{k}$ and problem (4) have the same minimum points.

Proof Let $S^{\star}$ be the set of vertices of $W \cap T$. Since the objective function of (5) is concave, and bounded below on $W \cap T$, by Proposition 1 it follows that $S^{\star}$ satisfies Assumption 1. Hence the thesis follows from Theorem 1.

Finally, consider the boolean problem (1) and the continuous formulation (3). The following result is immediate consequence of Proposition 2.

Corollary 1 Suppose that, for any $u \in U$, the objective function of problem (3) is concave and bounded below on the feasible set. Let $\left\{u^{k}\right\} \subset U$ be an infinite sequence such that Assumption 2 is satisfied. Then, for $k$ sufficiently large problem (3) with $u=u^{k}$ and problem (1) have the same minimum points.

\section{New penalty functions for zero-one concave programming}

In this section, we introduce new penalty functions depending on some parameters, and we show (using the general results stated in the previous section) that the penalty problems with continuous variables, for suitable values of the penalty function parameters, have the same global minimizers of the original zero-one programming problem (1). Assume that function $f$ of problem (1) is concave and bounded below on the relaxed feasible set

$$
\Omega=\{x \in P, 0 \leq x \leq e\} .
$$

Here are the concave formulations we propose:

\section{Formulation 1}

$$
\min _{x \in \Omega} f(x)+\sum_{i=1}^{n}\left\{\log \left(x_{i}+\epsilon\right)+\log \left[\left(1-x_{i}\right)+\epsilon\right]\right\}
$$

with $\epsilon>0$.

We observe that given $\epsilon$, the objective function is concave, bounded below on the feasible set. The equivalence between problem (13) and problem (1) is formally proved below.

Proposition 3 There exists a value $\bar{\epsilon}>0$ such that, for any $\epsilon \in(0, \bar{\epsilon}]$, problem (13) and problem (1) have the same minimum points. 
Proof Set $u=\epsilon$ and

$$
g(x, u)=\sum_{i=1}^{n}\left\{\log \left(x_{i}+\epsilon\right)+\log \left[\left(1-x_{i}\right)+\epsilon\right]\right\} .
$$

In order to prove the thesis, first we show that, given any sequence $\left\{u^{k}\right\}=\left\{\epsilon^{k}\right\}$ convergent to 0 , Assumption 2 holds for function $g\left(x, u^{k}\right)$. Then, we conclude, invoking Corollary 1 , that for $\epsilon$ sufficiently small (which corresponds to $k$ sufficiently large) problem (13) and problem (1) have the same minimum points.

For any $x \in X$ we have

$$
g\left(x, u^{k}\right)=n \cdot \log \left[\epsilon^{k} \cdot\left(1+\epsilon^{k}\right)\right] .
$$

So, recalling that $u^{k} \rightarrow 0$ for $k \rightarrow \infty$, we can write for each $\tilde{x} \notin X$ and for each $\bar{x} \in X$ :

$$
\begin{array}{r}
\lim _{k \rightarrow \infty}\left[g\left(\tilde{x}, u^{k}\right)-g\left(\bar{x}, u^{k}\right)\right]=\lim _{k \rightarrow \infty}\left\{\sum_{i=1}^{n} \log \left[\left(\tilde{x}_{i}+\epsilon^{k}\right) \cdot\left(1-\tilde{x}_{i}+\epsilon^{k}\right)\right]\right. \\
\left.-n \cdot \log \left[\epsilon^{k} \cdot\left(1+\epsilon^{k}\right)\right]\right\}=+\infty
\end{array}
$$

and Assumption 2 is satisfied.

Furthermore, for any $u \in U=R_{+}$, function (14) is concave and bounded below on the feasible set. Hence, by Corollary 1 , for any $k$ sufficiently large, problem (13) and problem (1) have the same minimum points.

\section{Formulation 2}

$$
\min _{x \in \Omega} f(x)+\sum_{i=1}^{n}\left\{-\left(x_{i}+\epsilon\right)^{-p}-\left[\left(1-x_{i}\right)+\epsilon\right]^{-p}\right\}
$$

with $\epsilon>0$ and $p \geq 1$.

We observe that, given $\epsilon$ and $p$, the objective function is concave, bounded below on the feasible set. The equivalence between problem (15) and problem (1) is formally proved below.

Proposition 4 There exists a value $\bar{\epsilon}>0$ such that, for any $\epsilon \in(0, \bar{\epsilon}]$, problem (15) and problem (1) have the same minimum points.

Proof Set $u=\epsilon$ and

$$
g(x, u)=\sum_{i=1}^{n}\left\{-\left(x_{i}+\epsilon\right)^{-p}-\left[\left(1-x_{i}\right)+\epsilon\right]^{-p}\right\}
$$


and let $\left\{u^{k}\right\}=\left\{\epsilon^{k}\right\}$ be the sequence convergent to 0 . For any $x \in X$ we have

$$
g\left(x, u^{k}\right)=-n \cdot\left[\left(\epsilon^{k}\right)^{-p}+\left(1+\epsilon^{k}\right)^{-p}\right] .
$$

So, recalling that $u^{k} \rightarrow 0$ for $k \rightarrow \infty$, we can write for each $\tilde{x} \notin X$ and for each $\bar{x} \in X$ :

$$
\begin{array}{r}
\lim _{k \rightarrow \infty}\left[g\left(\tilde{x}, u^{k}\right)-g\left(\bar{x}, u^{k}\right)\right]=\lim _{k \rightarrow \infty}\left\{\sum_{i=1}^{n}\left\{-\left(\tilde{x}_{i}+\epsilon^{k}\right)^{-p}-\left[\left(1-\tilde{x}_{i}\right)+\epsilon^{k}\right]^{-p}\right\}\right. \\
\left.+n \cdot\left[\left(\epsilon^{k}\right)^{-p}+\left(1+\epsilon^{k}\right)^{-p}\right]\right\}=+\infty
\end{array}
$$

and Assumption 2 is satisfied.

Furthermore, for any $u \in U=R_{+}$, and $p \geq 1$, function (16) is concave and bounded below on the feasible set. Hence, by Corollary 1 , for any $k$ sufficiently large problem (15) and problem (1) have the same minimum points.

\section{Formulation 3}

$$
\min _{x \in \Omega} f(x)+\frac{1}{\beta} \sum_{i=1}^{n}\left\{\left(1-\varepsilon^{-\frac{1}{\alpha} x_{i}}\right)+\left[1-\varepsilon^{-\frac{1}{\alpha}\left(1-x_{i}\right)}\right]\right\}
$$

with $\beta, \alpha>0$ and $\varepsilon$ the base of the natural logarithm.

We observe that given $\alpha$ and $\beta$, the objective function is concave, bounded below on the feasible set. The equivalence between problem (17) and problem (1) is formally proved below.

Proposition 5 There exist a value $\bar{\beta}>0$, such that, for any value $\beta \leq \bar{\beta}$, problem (17) and problem (1) have the same minimum points.

Proof Set $u=\beta$ and

$$
g(x, u)=\frac{1}{\beta} \sum_{i=1}^{n}\left\{\left(1-\varepsilon^{-\frac{1}{\alpha} x_{i}}\right)+\left[1-\varepsilon^{-\frac{1}{\alpha}\left(1-x_{i}\right)}\right]\right\}
$$

and let $\left\{u^{k}\right\}=\left\{\beta^{k}\right\}$ be the sequence convergent to 0 . For any $x \in X$ we have

$$
g\left(x, u^{k}\right)=\frac{n}{\beta^{k}}\left(1-\varepsilon^{-\frac{1}{\alpha}}\right)
$$


So, recalling that $u^{k} \rightarrow 0$ for $k \rightarrow \infty$, we can write for each $\tilde{x} \notin X$ and for each $\bar{x} \in X$ :

$$
\begin{gathered}
\lim _{k \rightarrow \infty}\left[g\left(\tilde{x}, u^{k}\right)-g\left(\bar{x}, u^{k}\right)\right]=\lim _{k \rightarrow \infty}\left\{\frac { 1 } { \beta ^ { k } } \cdot \left\{2 n-\sum_{i=1}^{n}\left[\varepsilon^{-\frac{1}{\alpha} \tilde{x}_{i}}+\varepsilon^{-\frac{1}{\alpha}\left(1-\tilde{x}_{i}\right)}\right]\right.\right. \\
\left.\left.-n \cdot\left(1-\varepsilon^{-\frac{1}{\alpha}}\right)\right\}\right\}=+\infty
\end{gathered}
$$

and Assumption 2 is satisfied.

Furthermore, for any $u \in U=R_{+}$, and $\alpha>0$, function (18) is concave and bounded below on the feasible set. Hence, by Corollary 1 , for any $k$ sufficiently large problem (17) and problem (1) have the same minimum points.

\section{Formulation 4}

$$
\min _{x \in \Omega} f(x)+\frac{1}{\beta} \sum_{i=1}^{n}\left[x_{i}^{p}+\left(1-x_{i}\right)^{p}\right]
$$

with $\beta>0$ and $0<p<1$.

We observe that, given $p$ and $\beta$, the objective function is concave, bounded below on the feasible set. The equivalence between problem (19) and problem (1) is formally proved below.

Proposition 6 There exist a value $\bar{\beta}>0$, such that, for any value $\beta \leq \bar{\beta}$, problem (19) and problem (1) have the same minimum points.

Proof Set $u=\beta$ and

$$
g(x, u)=\frac{1}{\beta} \sum_{i=1}^{n}\left[x_{i}^{p}+\left(1-x_{i}\right)^{p}\right]
$$

and let $\left\{u^{k}\right\}=\left\{\beta^{k}\right\}$ be the sequence convergent to 0 . For any $x \in X$ we have

$$
g\left(x, u^{k}\right)=\frac{n}{\beta^{k}} .
$$

So, recalling that $u^{k} \rightarrow 0$ for $k \rightarrow \infty$, we can write for each $\tilde{x} \notin X$ and for each $\bar{x} \in X$ :

$$
\lim _{k \rightarrow \infty}\left[g\left(\tilde{x}, u^{k}\right)-g\left(\bar{x}, u^{k}\right)\right]=\lim _{k \rightarrow \infty}\left\{\frac{1}{\beta^{k}} \cdot\left\{\sum_{i=1}^{n}\left[\tilde{x}_{i}^{p}+\left(1-\tilde{x}_{i}\right)^{p}\right]-n\right\}\right\}=+\infty
$$

and Assumption 2 is satisfied. 
Furthermore, for any $u \in U=R_{+}$, and $0<p<1$, function (20) is concave and bounded below on the feasible set. Hence, by Corollary 1 , for any $k$ sufficiently large problem (19) and problem (1) have the same minimum points.

Remark Two known continuous reformulations equivalent to (1) are:

- problem (2) proposed in [4];

- the following problem

$$
\min _{x \in \Omega} f(x)-\frac{1}{\beta} \sum_{i=1}^{n}\left(x_{i}-\frac{1}{2}\right)^{2}
$$

proposed in [7].

The general theoretical results stated in Sect. 2 allow us to easily reobtain the equivalence between the above continuous problems and the original 0-1 concave problem. We do not report the formal proofs for sake of brevity.

Acknowledgments The author is grateful to Prof. Marco Sciandrone of University of Florence for his useful comments and suggestions. The author would also like to thank the anonymous referee for his constructive comments and the comprehensive and detailed review of the article.

\section{References}

1. Abello, J., Butenko, S., Pardalos, P.M., Resende, M.: Finding independent sets in a graph using continuous multivariable polynomial formulations. J. Glob. Optim. 21, 111-137 (2001)

2. Balasundaram, B., Butenko, S.: Constructing test functions for global optimization using continuous formulations of graph problems. Optim. Methods Softw. 20, 439-452 (2005)

3. Borchardt, M.: An exact penalty approach for solving a class of minimization problems with Boolean variables. Optim. 19(6), 829-838 (1988)

4. Giannessi, F., Niccolucci, F.: Connections between nonlinear and integer programming problems. Symp. Math. 19, 161-176 (1976)

5. Horst, R., Pardalos, P.M., Thoai, N.V.: Introduction to Global Optimization, 2nd edn. Kluwer, Dordrecht (2000)

6. Kalantari, B., Rosen, J.B.: Penalty formulation for zero-one integer equivalent problem. Math. Program. 24, 229-232 (1982)

7. Kalantari, B., Rosen, J.B.: Penalty formulation for zero-one nonlinear programming. Discrete Appl. Math. 16(2), 179-182 (1987)

8. Mangasarian, O.L.: Machine learning via polyhedral concave minimization. In: Fischer, H., Riedmueller, B., Schaeffler, S. (eds.) Applied Mathematics and Parallel Computing-Festschrift for Klaus Ritter, pp. 175-188. Physica-Verlag, Germany (1996)

9. Mangasarian, O.L.: Knapsack Feasibility as an Absolute Value Equation Solvable by Successive Linear Programming. Data Mining Institute Technical Report 08-03, September 2008. Optimization Letters (2008, to appear)

10. Pardalos, P.M., Rosen, J.B.: Constrained Global Optimization: algorithms and Applications. Lecture Notes in Computer Science, vol. 268. Springer, Berlin (1987)

11. Pardalos, P.M., Prokopyev, O.A., Busygin, S.: Continuous Approaches for Solving Discrete Optimization Problems. Handbook on Modelling for Discrete Optimization, vol. 8, pp. 39-60. Springer, New York (2006)

12. Raghavachari, M.: On connections between zero-one integer programming and concave programming under linear constraints. Oper. Res. 17(4), 680-684 (1969) 
13. Rinaldi, F., Schoen, F., Sciandrone, M.: Concave programming for minimizing the zero-norm over polyhedral sets. Technical report RT 2/2008, Dipartimento Sistemi e Informatica, Università di Firenze. Computational Optimization and Applications (2008, to appear)

14. Rockafellar, T.: Convex Analysis. Princeton University Press, Princeton (1970)

15. Zhu, W.X.: Penalty parameter for linearly constrained $0-1$ quadratic programming. J. Optim. Theory Appl. 116(1), 229-239 (2003) 